\title{
VALIDAÇÃO DO APLICATIVO HELPTEEN PARA PREVENÇÃO DA VIOLÊNCIA CONTRA ADOLESCENTES
}

\author{
Maristela Inês Osawa Vasconcelos ${ }^{1}$ \\ Yanka Alcântara Cavalcante ${ }^{1}$ \\ Gabriel Pereira Maciel $^{1}$ \\ Milena Melo Vieira ${ }^{1}$ \\ Paulo Roberto Lopes ${ }^{1}$ \\ Joyce Mazza Nunes Aragão ${ }^{1}$ \\ Iane Ximenes Teixeira ${ }^{1}$ \\ Francisco Rosemiro Guimarães Ximenes Neto ${ }^{1}$
}

\begin{abstract}
https://orcid.org/0000-0002-1937-8850 https://orcid.org/0000-0003-0152-9216 https://orcid.org/0000-0002-4141-1995 https://orcid.org/0000-0002-8548-3755 https://orcid.org/0000-0003-0890-0612 https://orcid.org/0000-0003-2865-579X https://orcid.org/0000-0002-6790-6478 https://orcid.org/0000-0002-7905-9990
\end{abstract}

Objetivo: Validar o aplicativo móvel HelpTeen como tecnologia educativa para prevenção da violência contra adolescentes. Método: Trata-se de uma pesquisa de validação aplicada, com abordagem quantitativa, cuja coleta de dados aconteceu no mês de agosto de 2019 , em duas escolas públicas de ensino fundamental e médio de Sobral-Ceará. A validação de conteúdo e aparência foi realizada com 106 adolescentes de 14 a 18 anos. Os dados foram submetidos a estatística descritiva e expressos por meio do Índice de Validade de Conteúdo (IVC). Para fins de validação foi considerado o percentual mínimo de $75 \%$ de concordância para cada item. Resultados: os 17 itens de avaliação foram validados alcançando o referencial exigido com IVC>0,75, sendo avaliados com os menores indices, os itens: C $1.2(=0,77)$ sobre se as funções eram suficientes ao alcance de objetivos; o Cl.4 (=0,76) que trata do aprendizado de um novo conteúdo; C 2.3 (=0,77) quanto a quantidade apropriada de informação em cada tela; e D 1.3 (=0,77) sobre a exploração de conteúdos consistentes. Conclusão: Conclui-se que o aplicativo HelpTeen foi validado pelo público adolescente, embora ainda necessite aperfeiçoar algumas funções relacionadas ao aprendizado, como também no quesito da usabilidade visando maior interesse do aplicativo como ferramenta educativa para o público adolescente.

Descritores: Estudos de validação; Tecnologia da Informação; Ensino-aprendizagem. Saúde do Adolescente.

\section{VALIDATION OF THE HELPTEEN APPLICATION TO PREVENT VIOLENCE AGAINST ADOLESCENTS}

Objective: To validate the HelpTeen mobile application as an educational technology for preventing violence against adolescents. Method: This is an applied research, with a quantitative approach, whose data collection took place in August 2019 in two public elementary and high schools in Sobral-Ceará. The content and appearance validation was performed with 106 adolescents (14-18 years old). The data were submitted to descriptive statistics and expressed through the Content Validity Index (CVI). For validation purposes, the minimum percentage of $75 \%$ agreement for each item was considered. Results: the 17 evaluation items were validated reaching the required benchmark with CVI> 0.75, and the items were evaluated with the lowest indexes: $\mathrm{C} 1.2$ ( $=0.77)$ on whether the functions were sufficient to achieve objectives; o $\mathrm{Cl} .4$ (= 0.76 ) that deals with the learning of new content; $\mathrm{C} 2.3$ (= 0.77 ) regarding the appropriate amount of information on each screen; and D 1.3 ( $=0.77)$ on the exploration of consistent content. Conclusion: It is concluded that the HelpTeen application was validated by the adolescent public, although it still needs to improve some functions related to learning, as well as in terms of usability, aiming at the greater interest of the application as an educational tool for the adolescent public.

Descriptors: Validation studies; Information Technology: Teaching-learning; Adolescent Health.

\section{VALIDACIÓN DE LAAPLICACIÓN HELPTEEN PARA PREVENIR LA VIOLENCIA CONTRA LOS ADOLESCENTES}

Objetivo: validar la aplicación móvil HelpTeen como tecnología educativa para prevenir la violencia contra los adolescentes. Método: Esta es una investigación aplicada, con un enfoque cuantitativo, cuya recopilación de datos tuvo lugar en agosto de 2019, en dos escuelas públicas primarias y secundarias en Sobral-Ceará. La validación de contenido y apariencia se realizó con 106 adolescentes de 14 a 18 años. Los datos se enviaron a estadísticas descriptivas y se expresaron a través del Índice de Validez del Contenido (CVI). Para fines de validación, se consideró el porcentaje mínimo de $75 \%$ de acuerdo para cada artículo. Resultados: los 17 items de evaluación fueron validados alcanzando el punto de referencia requerido con CVI> 0.75, y los ítems fueron evaluados con los indices más bajos: C 1.2 (= 0.77) sobre si las funciones eran suficientes para lograr los objetivos; o Cl.4 (= 0.76) que se ocupa del aprendizaje de nuevos contenidos; C 2.3 (= 0.77) con respecto a la cantidad apropiada de información en cada pantalla; y D 1.3 (= 0.77 ) sobre la exploración de contenido consistente. Conclusion: Se concluye que la aplicación HelpTeen fue validada por el público adolescente, aunque todavía necesita mejorar algunas funciones relacionadas con el aprendizaje, asi como en términos de usabilidad, con el objetivo de un mayor interés de la aplicación como una herramienta educativa para el público adolescente.

Descritores: estudios de validación; Tecnología de la informacion; Enseñanza-aprendizaje. Salud del adolescente.

IUniversidade Estadual Vale do Acaraú - UVA, Sobral, CE, Brasil

Autor correspondente: Maristela Inês Osawa Vasconcelos - Email: miosawa@gmail.com

Recebido: 25/04/2020 - Aceito: 03/05/2020 


\section{INTRODUÇÃO}

O fenômeno da violência é um complexo problema de saúde pública por suas características polimórficas, multicausais, complexas, interligadas aos determinantes sociais e econômicos, como desemprego, exclusão social, baixa escolaridade, além dos aspectos culturais e comportamentais, como o racismo, o machismo e a homofobia ${ }^{1-2}$.

Apesar de os indices de criminalidade e atos violentos com adolescentes terem decaído em comparação à última década, o Estado do Ceará ainda apresenta uma das maiores taxas de mortes violentas com esse público, se comparados com outros estados, o que aponta a necessidade de serem desenvolvidas políticas públicas e ações intersetoriais, em especial aquelas relativas a educação em saúde, que busquem a promoção da cultura de paz e não violência ${ }^{3}$.

A realização das ações de promoção da saúde com a introdução de Tecnologias Digitais de Informação e Comunicação (TDIC) torna-se uma alternativa importante, que pode gerar impacto na prevenção aos diversos tipos de violência (Bullying, Cyberbullying, violência sexual, intrafamiliar, escolar, urbana, física, policial, o suicídio e o Abuso de Relacionamento Adolescente), a que estão expostos os adolescentes.

Ressalta-se que os estudantes do ensino fundamental, médio ou universitário, que estão na faixa etária da famosa "Geração Z", apresentam um elevado nivel de conectividade, o que facilita a disseminação de conteúdos de educação em saúde em diferentes aspectos e temas no formato de aplicativos ${ }^{4}$.

O uso da TDIC em associação com temáticas referentes à saúde têm obtido resultados efetivos, por romper com barreiras, como a timidez, o receio e às vezes a discriminação, que dificultam os adolescentes a ter um contato maior com os serviços de saúde. Além do mais, o uso das tecnologias como ferramenta pedagógica possibilita a aprendizagem ampliada, diversificada, dinâmica e significativa que favorece tanto adolescentes quanto profissionais da saúde ${ }^{5-6}$

O uso de tecnologias para educação em saúde pela Enfermagem têm crescido exponencialmente, ao passo que os casos clínicos e simulações são considerados os melhores exemplos para reprodução do processo de ensino-aprendizagem em profissionais da área e determinadas populações ${ }^{7}$.
Desta forma, o presente estudo teve por objetivo validar o aplicativo móvel HelpTeen como tecnologia educativa para prevenção da violência contra adolescentes.

\section{MÉTODO}

\section{Tipo de Estudo}

Pesquisa aplicada, que visa a validação ${ }^{8}$ de um aplicativo móvel.

\section{Local do Estudo}

O estudo ocorreu em duas escolas públicas de ensino fundamental e médio de Sobral - Ceará, município com uma população estimada para o ano de 2019 de 208.935 habitantes 9.

\section{Participantes do Estudo}

A população do estudo foi constituída de 106 estudantes do ensino médio. Para seleção dos participantes, adotaram-se os seguintes critérios: ter idade entre 14 e 19 anos, estar regularmente matriculado entre o primeiro e o terceiro ano, ter autorização dos pais e/ou responsáveis por meio da assinatura do Termo de Consentimento Livre e Esclarecido (TCLE) ou o Termo de Assentimento Livre e Esclarecido (TALE) e possuir aparelho de telefonia móvel compatível com o aplicativo androide. Os critérios de exclusão foram: não comparecer à escola no dia da coleta dos dados e/ou apresentar algum comprometimento que pudesse inviabilizar a avaliação do aplicativo.

\section{Coleta de Dados}

A coleta de dados ocorreu no mês de agosto de 2019, nos três turnos, sendo solicitado aos estudantes $(\mathrm{N}=106)$ que fizessem o download do aplicativo móvel HelpTeen (Figura 1). Após download, as funcionalidades foram apresentadas e foi concedido um tempo de 30 minutos para que pudessem utilizar o aplicativo, sendo fornecidas explicações diante das dúvidas. $O$ instrumento de avaliação foi adaptado do original desenvolvido por Batista ${ }^{10}$ composto por cinco blocos (A, B, C, D e E) e suas subdivisões, com 109 questões. Deste, dois blocos, o C e D, foram adaptados com 17 questões para validar o HelpTeen. Para cada item avaliado, têm-se uma escala do tipo Likert com seis opções (Sim; Parcialmente com poucas restrições; Parcialmente; Parcialmente com muitas restrições; Não, e Não se aplica). 
Figura 1 - Logotipo, tela inicial e tela da função "Bate Papo" do aplicativo HelpTeen. Sobral - Ceará, Brasil, 2019.

\section{6 \\ HELPTEEN}

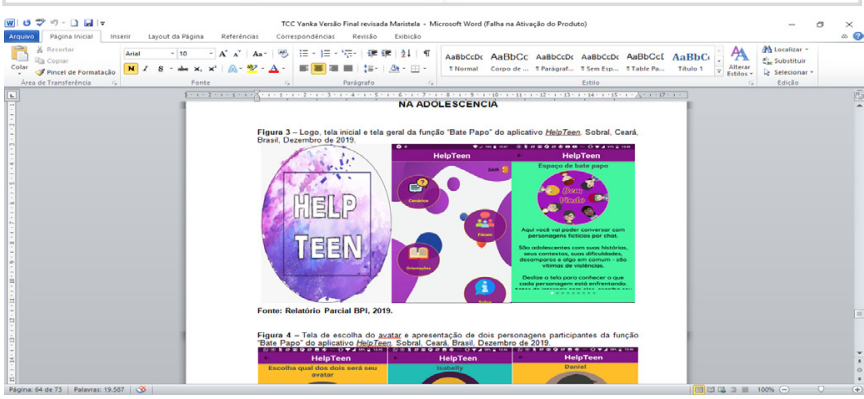

Procedimentos de Análise e Tratamento das Informações

Após o acesso e utilização do aplicativo os adolescentes tiveram tempo de 30 minutos para avaliação deste.

Os dados foram compilados em planilhas e analisados com o apoio do software Statistical Package for the Social Sciences (SPSS) versão 23.0 para Windows. A análise descritiva da validação envolveu cálculos de frequências absolutas e relativas para variáveis nominais.

Para análise da validação de conteúdo e aparência dos componentes do aplicativo utilizou-se o Índice de Validação de Conteúdo (IVC), o qual afere a concordância do público alvo à representatividade dos itens em relação ao conteúdo em estudo, sendo eles, Individual (I-IVC), (S-IVC/ Ave) média dos conjuntos e Global (S-IVC/Global), medindo a proporção ou porcentagem de concordância de juízes, neste caso, o público-alvo, sobre determinados aspectos de uma tecnologia e seus domínios de conhecimento para avaliação ${ }^{11}$

Para calcular o IVC de cada item do instrumento, foi realizada a soma das respostas "quatro" (Parcialmente com Poucas Restrições) e "cinco" (Sim) dos participantes e dividido o resultado dessa soma pelo número total de respostas, conforme fórmula a seguir: IVC = Número de respostas "quatro" ou "cinco"/Número total de respostas".

O IVC > 0,75 foi adotado como critério, onde cada item de avaliação foi considerado válido somente quando acima deste valor ${ }^{11-14}$.

\section{Aspectos Éticos}

Esta pesquisa respeitou os princípios éticos que visa assegurar os direitos e deveres da comunidade científica, dos participantes da pesquisa e da sociedade ${ }^{12}$. Este estudo faz parte de uma pesquisa maior, intitulada "Aplicativo multimídia HelpTeen em plataforma móvel como tecnologia educativa para prevenção da violência contra adolescentes: Construção e Validação", que foi submetido ao Comitê de Ética e Pesquisa (CEP) sendo aprovado sob Parecer $n$ 의 3.273 .823$.

\section{RESULTADOS}

A Tabela 1 - apresenta os dados de IVC individual, Média e Global quanto à avaliação do aplicativo HelpTeen.

Tabela 1 Distribuição dos Índices de Validação de Conteúdo dos elementos do aplicativo conforme a análise dos adolescentes escolares. Sobral - Ceará, Brasil, 2019.

\begin{tabular}{|c|c|c|}
\hline Itens & I-IVC & $\begin{array}{l}\text { S-IVC/ } \\
\text { Ave }\end{array}$ \\
\hline
\end{tabular}

BLOCO C - Caracteristicas pedagó-

gicas gerais

0,85

Objetivos (Cl)

0.83

C 1.1 Acha que os objetivos ficaram claros?

0,96

C 1.2 As funções são suficientes para

alcance do objetivo?

C 1.3 Desperta o interesse dos adoles-

centes sobre o assunto?

C 1.4 Aprendeu algum conteúdo novo utilizando o aplicativo?

Usabilidade/Interface (C2) 0,87

C 2.1 A forma que o aplicativo é apre-

sentado (cores, imagens, textos) está

0,86

adequado?

C 2.2 Funções são fáceis de serem

utilizadas?

0,98

C 2.3 A quantidade de informação em

cada tela é apropriada?

C 2.4 As mensagens são claras e

fáceis de serem entendidas?

c 2.5 Animação e outras mídias são

2.5 Animação e outras mídias são $\quad 0,84$

agradáveis?

C 2.6 As cores são utilizadas com equilibrio, ou seja, são bem distribui0.83

das evitando poluição visual? 
C 2.7 É ofertado meio(s) para apresen-

tar sugestões e/ou recomendações?

$\begin{array}{ll}\text { BLOCO D - Avaliação do Software } & 0,86 \\ \text { segundo sua Proposta Educacional } & \end{array}$

D 1.1 A forma como o aplicativo foi

organizado possui uma lógica interna

desafiadora, porém fácil de ser domi-

0,86

nada pelo usuário?

\begin{tabular}{|c|c|}
\hline $\begin{array}{l}\text { D } 1.2 \text { Explora os conteúdos de maneira } \\
\text { lúdica? }\end{array}$ & 0,81 \\
\hline $\begin{array}{l}\text { D } 1.3 \text { Explora os conteúdos de forma } \\
\text { consistente, ou seja, não apenas } \\
\text { superficialmente? }\end{array}$ & 0,77 \\
\hline $\begin{array}{l}\text { D 1.4 Pode motivar o questionamento } \\
\text { sobre o assunto? }\end{array}$ & 0,92 \\
\hline $\begin{array}{l}\text { D } 1.5 \text { Oferece reforço positivo em } \\
\text { momentos adequados? }\end{array}$ & 0,94 \\
\hline $\begin{array}{l}\text { D } 1.6 \text { Quando o aplicativo solicita que } \\
\text { execute atividades, você acha que ele } \\
\text { faz de maneira clara? }\end{array}$ & 0,87 \\
\hline
\end{tabular}

De acordo com o percentual mínimo exigido, todos os itens de avaliação do aplicativo $(n=17)$ obtiveram I-IVC > 0,75 pelos 106 adolescentes, o que condiz com o eficaz planejamento e levantamento de requisitos para construção do sistema hipermídia, não havendo a necessidade de uma segunda rodada de validação.

O IVC Global $(=0,85)$ obtido foi validado de acordo com o referencial adotado e quanto ao S-IVC/Ave dos conjuntos, os blocos C e D atingiram IVC>0,80; sendo o Bloco C (Características pedagógicas gerais) com IVC igual a 0,85; Objetivos (Cl) com IVC igual a 0,83; e Usabilidade/Interface igual a 0,87 e o Bloco D (Avaliação do Software segundo sua Proposta Educacional) com 0,86, o que designa bom desempenho de avaliação da média do aplicativo HelpTeen.

Um comentário positivo no item Cl.l (Os objetivos ficaram claros?) com IVC de 0,96 descreveu o aplicativo como uma tecnologia com ótimo objetivo e inovador onde a ideia principal tende a apoiar vários adolescentes de diferentes faixas etárias.

$\mathrm{O}$ item $\mathrm{Cl} .3$ (Desperta o interesse dos adolescentes sobre o assunto?) obteve IVC $(=0,83)$, tendo como elogio que o aplicativo era bom e podia despertar o interesse de outros jovens.

O item Cl.2 (As funções são suficientes para o alcance do objetivo?) apresentou I-IVC de 0,77, sendo um dos itens com menor índice de validade, com crítica à respeito das funções do aplicativo apresentarem-se como suficientes aos objetivos de prevenir à violência por meio da educação em saúde.

O item Cl.4 (Aprendeu algum conteúdo novo utilizando o aplicativo?) obteve I-IVC de 0,76 tornando-se o menor indice de validade dentre os 17 itens, no qual deve-se ser revisto as funções e ideias refletidas na tecnologia para a devida melhoria.

O item C2.2 (Funções são fáceis de serem utilizadas?) apresentou I-IVC de 0,98, tornando-se o maior índice do estudo, representando a relevância da implantação de funções que possam ser fáceis ao entendimento do público em questão, assim como a necessidade de desenvolvimento de futuras tecnologias, sobretudo aquelas relacionados à aplicativos de plataforma Web e para Android/iOS, que tenham funcionalidade compreensivel ao olhar crítico e explorador do adolescente.

Os itens C 2.4 (As mensagens são claras e fáceis de serem entendidas?) obtive I-IVC de 0,94 de concordância pelo público, onde para que exista a compreensão do reforço positivo ofertado pelo aplicativo, é necessário que as mensagens estejam totalmente concisas e fáceis ao nivel de experiência dos adolescentes.

O item C2.7 (É ofertado meio(s) para apresentar sugestões e/ou recomendações?) alcançou I-IVC de 0,89, conforme é apresentado pela parte condicionada ao Sobre, em que os comentários visualizados de elogios mencionaram que o adolescente consegue visualizar a equipe técnica do projeto, os agradecimentos e a função de sugestões para melhorias do aplicativo.

Quanto ao item C2.1 (A forma que o aplicativo é apresentado, cores, imagens e textos, está adequado?) apresentou I-IVC de 0,86, indicando excelente nivel de adequação contudo apresentou dois comentários sugestivos à melhora do aplicativo, mudando as cores, deixando-as "mais vivas", estando bastante saturadas, sendo recomendado a suavização e um efeito gradiente. Outros comentários afirmaram a necessidade de ter uma linguagem e texto que estivesse no dia-a-dia dos jovens, com a adição de girias, que estivessem menos normalizados e repetitivos, a fim de melhorar ambiente e torná-lo familiar e confortável.

O item C2.5 (Animação e outras mídias são agradáveis?) obteve I-IVC de 0,84 com ótimo rendimento quanto às animações implementadas ao aplicativo, tendo comentários quanto a excelente utilidade e ao ambiente agradável, assim como a necessidade de melhoria do design com melhores animações e mais explicações, além de modificar o "Bate Papo", deixando-o extenso no quesito de conversa, anteriormente mencionado e em fase de revisão. 
O item C2.6 (As cores são utilizadas com equilibrio, ou seja, são bem distribuídas evitando poluição visual?) alcançou I-IVC de 0,83, apresentando os mesmos comentários dos adolescentes listados ao item C2.1 (A forma que o aplicativo é apresentado, cores, imagens e textos, está adequado?), em que necessita de uma nova abordagem das cores utilizadas nas funções do aplicativo, como forma de melhorar a harmonia do espaço para atração visual do público.

Concluindo o bloco $\mathrm{C}$ quanto aos itens avaliados para a tecnologia HelpTeen, têm-se o item C2.3 (A quantidade de informação em cada tela é apropriada?) com I-IVC de 0,77 tornando-se o menor índice de validade referente ao sub-bloco de Usabilidade/Interface.

O item D1.5 (Oferece reforço positivo em momentos adequados?) alcançou o maior índice de validade do bloco com I-IVC>0,90; obtendo excelente resposta do público adolescente às situações implantadas pelo aplicativo que ofertam o devido reforço ao estímulo do estudo sobre a violência, quais os tipos e quais as redes de enfrentamento para esta.

O item Dl.4 (Pode motivar o questionamento sobre o assunto?) atingiu I-IVC de 0,92, tendo excelente resposta de validade do público adolescente, permitindo a análise da motivação quanto ao questionamento dos tipos de violência, locais em que mais ocorrem e as estratégias de enfrentamento, assuntos utilizados e incentivados pelo aplicativo HelpTeen

O item D1.6 (Quando o aplicativo solicita que execute atividades, você acha que ele faz de maneira clara?) apresentou I-IVC de 0,87 com bom desempenho de acordo com as funções e mensagens empenhadas pelo aplicativo sobre as atividades solicitadas aos adolescentes. Não houve comentários referentes à este item.

O item Dl.1 (A forma como o aplicativo foi organizado possui uma lógica interna desafiadora, porém fácil de ser dominada pelo usuário?) alcançou I-IVC satisfatório de 0,86 como concordância do público-alvo, representando boa lógica desempenhada pelo aplicativo em desafiar o adolescente no aprofundamento sobre a violência em seus diversos meios e aspectos.

Quanto ao item D1.2 (Explora os conteúdos de maneira lúdica?) obteve I-IVC de 0,81, tornando-se o segundo menor do bloco D, havendo a necessidade de modificar algumas das funções impostas ao aplicativo para uma forma cômica e atraente ao olhar do adolescente.

Encerrando-se o bloco D, têm-se o item Dl.3 (Explora os conteúdos de forma consistente, ou seja, não apenas superficialmente?) com menor índice de validação pelo bloco, tendo obtido I-IVC de 0,77 sendo repensado quanto à consistência das funções que trazem os conteúdos referentes à violência na adolescência.

\section{DISCUSSÃO}

A validação do aplicativo, apontou um IVC/Global > 0,80 com 85\% de concordância total dos 106 adolescentes, o que indica uma avaliação satisfatória do HelpTeen.

Os conhecimentos teóricos e práticos associados à informática em âmbito educacional têm repercutido amplamente a nivel mundial, pois as mídias digitais oferecem à didática, instrumentos capazes de renovar as situações de interação, expressão, criação, comunicação, informação, e colaboração, tornando-a muito diferente daquela tradicionalmente fundamentada na escrita e em meios impressos ${ }^{13}$

Corroborando com o dado encontrado, estudos semelhantes ${ }^{14-15}$ à validação de tecnologias educativas para adolescentes também validaram seus materiais com indices de validade satisfatórios com IVC global variaram entre 0,97 a 0,75.

A tecnologia educativa obteve destaque em decorrência ao contexto cultural em que se encontra a contemporaneidade, no qual a formação do adolescente tem exigido, não somente conhecimentos teóricos, pensamentos reflexivos e críticos, como também a mudança pelas transformações de sua realidade social de acordo com os recursos disponibilizados ${ }^{16}$.

Materiais educativos, advindos das diversas formas de repercussão, sejam por cartilhas, manuais e/ou AVA (Websites, Aplicativos móveis), que são avaliados por instrumentos de conteúdo à área da saúde, facilitam o processo de trabalho de profissionais por meio da orientação de terminado tipo de público-alvo, a exemplo de adolescentes, no qual possuem a finalidade de desenvolver ações de promoção da saúde, com garantia efetiva do cuidado destes, sendo então, o item Cl.l (Acha que os objetivos ficaram claros?) pertinente à compreensão do conteúdo que será repassado quanto à violência na adolescência ${ }^{17}$

$O$ adolescente encontra-se em sua subjetividade, sendo uma estrutura dinâmica com a capacidade de reconstrução do pensamento e das opiniões em meio aos contextos em que se insere, o que pode embasar as escolhas de respostas diferenciadas ao item Cl.3 (Desperta o interesse entre os jovens?) $)^{18}$.

Em relação ao item $\mathrm{Cl}$.2 (As funções são suficientes para o alcance do objetivo?), compreende-se que uma única tecnologia educativa não seria capaz de fornecer todo o alcance de prevenção à violência como estratégia de edu- 
cação em saúde, devendo haver políticas públicas inclusivas, que promovam a cultura de paz e não violência.

A violência antes de ser um problema intelectual, configura-se por uma questão social e política, podendo afetar materialmente os serviços de saúde e levantar custos, devendo ser acompanhada por estratégias intersetoriais $^{19}$. Por sua determinação social, magnitude e amplitude, cada vez mais a violência se configura como uma demanda constante dos serviços de saúde, e para seu desvelamento, necessita de ações de educação em saúde na comunidade, para que ocorra uma efetiva prevenção e identificação de casos.

O item C2.2 (Funções são fáceis de serem utilizadas?) foi validado com IVC de 0,98, similar à validação de um aplicativo para prevenção à incontinência urinária em mulheres no pós-parto ${ }^{20}$ que descreve dados de IVC parecidos, em que os itens de funcionalidade fácil para compreensão e usabilidade apresentaram os I-IVC de 0,90 e 1 (validação total pelo índice máximo), respectivamente, o que determina impacto positivo no planejamento prévio da funcionalidade dos aplicativos diante da sua facilidade de uso e adaptação daqueles que estarão em contato e a importância da inserção das Ciências da Computação no diferencial do desenvolvimento prático e objetivo da tecnologia.

O material educativo quando bem elaborado deve dar suporte para a promoção de um espírito crítico, tornando certos discursos explícitos, ao invés de reproduzir representações negativas e acríticas com base em posturas e sistemas discursivos hegemônicos da sociedade ${ }^{21}$.

A inovação do processo educacional pelo ingresso de novas tecnologias educativas reflete a inovação do ensino-aprendizagem, uma vez que refere-se aos ideais de adaptação dos modelos existentes na educação contemporânea e traz-se a relevância de tais tecnologias à prática clínica voltada ao público adolescente, no qual os profissionais da saúde - em especial, a enfermagem pelas atribuições de promoção e prevenção da saúde- terão vantagens no aprimoramento de suas habilidades sendo bem manuseadas, permitindo o impacto positivo nos cuidados necessários ao público em questão ${ }^{22}$.

Diante do item C2.1 (A forma que o aplicativo é apresentado - cores, imagens e textos - está adequado?) com IVC de 0,86, um estudo similar com I-IVC de 0,82 de concordância dos juizes e cinco adolescentes aprovaram a tecnologia educacional perante as características de apresentação do jogo como tamanho e estilo da letra, cores, entre outros, havendo a motivação em estar no jogo do início ao fim, o que torna tais características cruciais em alinhamento para atração do público adolescente em man- ter-se participante da tecnologia, produzindo experiências e melhorando seus conhecimentos nas áreas situadas à adolescência $^{14}$.

Pelo item C2.5 (Animações e outras mídias são agradáveis?) com IVC < de 0,85, 8 destacam alguns pontos cruciais para que um aplicativo seja atraente aos olhos do consumidor, sendo estes: a interatividade (por diversas maneiras, com múltiplos toques, o arrastar de dedos, os movimentos); a conectividade (interação e compartilhamento de informações por meio das redes sociais; integração (habilidade de obtenção dos dados de contatos); visual elegante (belas animações e alta resolução); sons e vídeos como fundamentais; e a velocidade, no qual lentidão contribuem exponencialmente para o descrédito da tecnologia6.

É importante ressaltar que o aplicativo HelpTeen não possui sons e vídeos, o que pode influenciar em uma avaliação menos efetiva ao passo que adolescentes se sentem atraídos por estes e tendem a fazer maior uso quando há a existência no sistema multimídia ${ }^{18}$.

Quanto ao item Dl.5 (Oferece reforço positivo em momentos adequados?), ambientes educacionais virtuais transvertidos que fornecem uma participação relevante nesse discurso educacional e podem servir como apoio para que professor pratique a avaliação formativa. Reitera-se aqui que um dos princípios subjacentes da avaliação formativa é fornecer feedback útil e utilizável ${ }^{23}$.

Faz-se necessária produção de tecnologias que transcendam o tecnicismo e a mecanização do trabalho. As TDIC devem estar inseridas na práxis do adolescente sob uma perspectiva pedagógica que alia o cuidar e educar em saúde, objetivando proporcionar aos indivíduos uma possibilidade para o desenvolvimento da crítica, construção/fortalecimento do conhecimento, bem como aprendizagem significativa ${ }^{24}$.

Soma-se a este contexto a motivação no processo de ensino/aprendizagem, devendo ser presente em todas as situações ofertadas ${ }^{25}$. A escola torna-se agente influenciador por facilitar, pelos professores, o processo de formação, no qual será influenciador à motivação da aprendizagem significativa, perante a ideia repassada pelo item Dl.4 (Pode motivar o questionamento sobre o assunto?).

A utilização de metodologias ativas, enquanto ferramentas pedagógicas de ensino de adolescentes em um contexto geral favorece o processo de ensino-aprendizagem, no qual realiza efetivamente a vivência de forma antecipada a prática e à teoria, por meio da busca por solução de problemas de saúde comuns ${ }^{26}$.

O item Dl.3 (Explora os conteúdos de forma consistente?), traz importante questionamento destacando o valor da educação problematizadora, aquela que respeita e valo- 
riza a criatividade, o pensamento crítico e o conhecimento trazido pelo estudante ao ambiente educativo ${ }^{27}$.

Ressalta-se, ainda, que as funcionalidades presentes no aplicativo têm o objetivo de intensificar o processo de aprendizado quanto à violência no contexto juvenil, podendo também despertar o interesse de diferentes profissionais para o uso e novas produções de tecnologias educacionais ${ }^{28-30}$, permitindo o alinhamento do conhecimento teórico-prático sobre o tema central ${ }^{29}$ e a busca de melhorias na qualidade da assistência de adolescentes vítimas de violência ${ }^{31}$.

Espera-se que este aplicativo possa ser utilizado como recurso para promoção de conhecimentos sobre a temática da violência, como também oportunize o empoderamento de adolescentes frente às situações de violência, possibilitando a reflexão sobre as estratégias de enfrentamento a serem tomadas quando da ocorrência, bem como enfatize a relevância das redes de apoio.

\section{Limitações do estudo}

Destaca-se como uma limitação o fato da maioria dos adolescentes não ter conseguido fazer o download do aplicativo e explorar suas funcionalidades com antecedência à avaliação, por não dispor de internet em seus lares, tendo acesso somente no ambiente escolar. O pouco tempo para apreciar o aplicativo pode trazer algum viés. No entanto, a superação dessas limitações exigirá um método de coleta de informações que envolva mais tempo e trabalho mais intenso.

\section{Contribuições para a prática}

O desenvolvimento de aplicativos para fins educativos é uma tendência contemporânea e cabe às universidades, bem como aos profissionais que atuam nas áreas educacional e/ou clínico-assistencial da saúde, apropriarem-se dessas metodologias e testarem nas áreas de atuação, de modo a verificarem a efetividade do potencial educativo destas estrategias.

\section{CONCLUSÃO}

Após passar por um processo rigoroso de validação, o aplicativo móvel HelpTeen foi validado pelos adolescentes. Todavia deve-se considerar que aprimoramentos podem ser realizados diante das sugestões do processo de avaliação, algumas ilustrações e textos devem ser ajustados a fim de torná-lo mais atraente e eficaz.

\section{Contribuições dos autores}

Agradecimenbtos: À Fundação Cearense de Apoio ao Desenvolvimento Científico e Tecnológico (FUNCAP) pelo financiamento da pesquisa, Edital de $n^{\circ}$. 03/2018 - Programa de Bolsas de Produtividade em Pesquisa, Estímulo à Interiorização e Inovação Tecnológica (BPI). Ao Laboratório de Pesquisa Social, Educação Transformadora e Saúde Coletiva - LABSUS, por ser espaço de reflexão crítica, conhecimento e afetividade.

\section{REFERÊNCIAS}

1. Ministério da Saúde (BR). Prevenção de violências e promoção da cultura de paz. Brasilia: Ministério da Saúde; 2008. [cited 2020 Apr 12]. Available from: http://bvsms.saude.gov.br/bvs/publicacoes/impacto_violencia_saude_criancas.pdf

2. Malta DC, Bernal RTI, Pugedo FSF, Lima Cheila Marina, Mascarenhas Marcio Denis Medeiros, Jorge Alzira de Oliveira et al. Violências contra adolescentes nas capitais brasileiras, segundo inquérito em serviços de urgência. Ciênc. saúde coletiva [Internet]. 2017 Sep [cited 2020 Apr 12]: 22(9): 2899-2908. Available from: http://www.scielo.br/scielo. php?script=sci_arttext\&pid=S1413-81232017002902899\&lng=en. https://doi.org/10.1590/1413-81232017229.14212017

3. Ceará. Assembleia Legislativa. Cada Vida Importa. Relatório final do Comitê Cearense pela Prevenção de Homicidios na Adolescência. Fortaleza: Assembléia Legislativa; 2016. [cited 2020 Apr 12]. Available from: https://www.al.ce.gov.br/phocadownload/relatorio_final.pdf.

4. Buckingham D. Cultura digital, educação midiática e o lugar da escolarização. Educação e Realidade. [Internet]. 2010 [cited 2020 Apr 12]: 35(3): 37-58. Available from: :http://www.seer.ufrgs.br/index.php/ educacaoerealidade/article/view/13077/10270.

5. Aragão JMN, Gubert FA, Torres RAM, Silva ASR, Vieira NFC. O uso do Facebook na aprendizagem em saúde: percepções de adolescentes escolares. Rev. Bras. Enferm. [Internet]. 2018 Apr [cited 2020 Apr
12]; $71(2):$ 265-271. Available from: http://www.scielo.br/scielo.php?script=sci_arttextEpid=S0034-71672018000200265\&lng=en. https:// doi.org/10.1590/0034-7167-2016-0604.

6. Pereira FG. Silva DV, Sousa LM, Frota NM. Building a digital application for teaching vital signs. Rev Gaúcha Enferm [Internet]. 2016 [cited 2020 Apr 19]:37(2):e59015. Available from: http://www.scielo.br/pdf/ rgenf/v37n2/en_0102-6933-rgenf-1983-144720160259015.pdf.

7. Millão LF, Vieira TW, Santos ND, Silva APSS, Flores CD. Integration of digital technologies in nursing teaching: simulation of a clinical case about pressure ulcers with the SIACC software. Reciis [Internet]. 2017 [cited 2020 Apr 20]:11(1):1-12. Available from: http://www.brapci.inf. br/ v/a/23038

8. Coluci MZO, Alexandre NMC, Milani D. Construção de instrumentos de medida na área da saúde. Ciênc. saúde coletiva [Internet]. 2015 Mar [cited 2020 Apr 12]; 20(3):925-936. Available from: http://www.scielo.br/ scielo.php?script=sci_arttext\&pid=S1413=81232015000300925-Elngen. https://doi.org/10.1590/1413-81232015203.04332013

9. Instituto Brasileiro de Geografia e Estatistica (IBGE). População estimada para o ano de 2019. [cited 2020 Apr 12]. Available from: https:// cidades.ibge.gov.br/brasil/ce/sobral/panorama.

10. Batista SCF. Softmat: um repositório de softwares para matemática do ensino médio - um instrumento em prol de posturas mais cons- 
cientes na seleção de softwares educacionais. Campos dos Goytacazes/RJ, Dissertação (Mestrado em Ciências da Engenharia) Centro de Ciência e Tecnologia da Universidade Estadual do Norte Fluminense. 2004, 202p. [cited 2020 Apr 12]. Available from: http://www.geogebra. im- uff.mat.br/biblioteca/dissertacao-batista-2004.pdf.

11. Lima ACMAC, Bezerra KC, Sousa DMN Rocha, JFreitas, Oriá MOB. Construção e Validação de cartilha para prevenção da transmissão vertical do HIV. Acta paul. enferm. [Internet]. 2017 Apr [cited 2020 Apr 12]; 30(2): 181-189. Available from: http://www.scielo.br/ scielo.php?script=sci_arttext\&pid=S0103=21002017000200181-\&lngen. https://doi.org/10.1590/1982-0194201700028.

12. Ministério da Saúde (BR). Resolução № 466/2012, de 13 de junho de 2013 do Conselho Nacional de Saúde/MS sobre Diretrizes e Normas Regulamentadoras de pesquisa envolvendo seres humanos. Diário Oficial da União. 2012. [cited 2020 Apr 12]. Available from: http://conselho. saude.gov.br/resolucoes/2012/Reso466.pdf.

13. Sousa RP, Miota FMCSC, Carvalho, ABG., orgs. Tecnologias digitais na educação [online]. Campina Grande: EDUEPB; 2011. 276 p. Available from: http://books.scielo.org.

14. Feitosa MCR, Stelko-Pereira ACC, Matos KJN. Validation of Brazilian educational technology for disseminating knowledge on leprosy to adolescents. Rev. Bras. Enferm. [Internet]. 2019 Oct [cited 2020 Apr 12]; 72(5):1333-1340. Available from: http://www.scielo.br/ scielo.php?script=sci_arttextEpid=\$0034=71672019000501333-\&lngen. Epub Sep 16, 2019. https://doi.org/10.1590/0034-7167-2018-0610.

15. Moura JR, Silva KC, Rocha AE, Santos SD, Amorim TR, Silva AR. Construção e validação de cartilha para prevenção do excesso ponderal em adolescentes. Acta paul. enferm. [Internet]. 2019 Aug [cited 2020 Apr 12]: 32(4):365-373. Available from: http://www.scielo.br/scielo.php?script=sci_arttext\&pid=S0103=21002019000400365-\&lngen. Epub Aug 12, 2019. https://doi.org/10.1590/1982-0194201900051.

16. Alarcon MFS, Prezotto KH. Evaluation of educational strategy, grounded on problem based learning on nursing undergraduate. Rev Rene. [Internet]. 2016 [cited 2020 Apr 12]; 17(2). Available from: http:// www.redalyc.org/html/3240/324045343012/.

17. Leite SS, Áfio ACE, Carvalho LV, Silva JM, Almeida PC, Pagliuca LMF. Construction and validation of an Educational Content Validation Instrument in Health. Rev. Bras. Enferm. [Internet]. 2018 [cited 2020 Apr 12]; 71( Suppl 4):1635-1641. Available from: http://www.scielo.br/scielo. php?script=sci_arttextEpid=\$0034-71672018001001635\&lng=en. https://doi.org/10.1590/0034-7167-2017-0648.

18. Oliveira MCSP. O adolescente em desenvolvimento e a contemporaneidade. In: Sudbrack MFO, Conceição MIG, Costa LF. (Org.). Curso de prevenção do uso de drogas para educadores de escolas públicas - edição revisada. 5ed. Brasilia: Secretaria Nacional De Políticas Sobre Drogas; 2012. v. 1. p. 30-37. [cited 2020 Apr 12]. Available from: http://www.aberta.senad.gov.br/medias/original/201704/20170424-094551-001.pdf

19. Minayo MCS. Conceitos, teorias e tipologias da violência: a violência faz mal a saúde. In: Njaine K, Assis K, Assis SG, Constantino P. Impactos da Violência na Saúde. FIOCRUZ. 2007; 1- 420. [cited 2020 Apr 12]. Available from: http://books.scielo.org/id/7yzrw/pdf/njaine-9788575415887.pdf.

20. Saboia DM. Construção e Validação de Aplicativo Educativo para Prevenção da Incontinência Urinária em Mulheres após o Parto. 2017. 150f. Dissertação (Mestrado em Enfermagem) - Faculdade de Farmá- cia, Odontologia e Enfermagem. Universidade Federal do Ceará, Fortaleza, 2017. [cited 2020 Apr 12]. Available from: : http: //www.repositorio.ufc.br/handle/riufc/29100.

21. Góes FSN, Fonseca LMM, Camargo RAA, Oliveira JF, Felipe HF. Educational technology "Anatomy and Vital Signs": Evaluation study of content, appearance and usability. J Med Inform. [Internet]. 2015 [cited 2020 Apr 12]; 84(11):982-987. Available from: http://www.sciencedirect.com/science/article/pii/S1386505615300149.

22. Holanda VR, Pinheiro AKB. Development of a hypermedia system for interactive e teaching of sexually transmitted diseases. Rev. enferm. UFPE on line. [Internet]. 2015 [acesso em 19 abr 2020]: 9(Suppl 2). Available from: https://periodicos.ufpe.br/revistas/revistaenfermagem/article/viewFile/10400/11165

23. Barab S, Sadler T, Heiselt C, Hickey D, Zuiker S. Relating narrative, inquiry, and inscriptions: a framework for socioscientific inquiry Journal of Science Education and Technology. [Internet]. 2007 [cited 2020 Apr 12]; 16(1):59-82. Available from: https://link.springer.com/ article/10.1007/s10956-006-9033-3.

24. Lanzoni GMM, Magalhães ALP, Costa VT, Erdmann AL, Andrade SR, Meirelles BHS. Tornando-se gerente de enfermagem na imbricada e complexa fronteira das dimensões assistencial e gerencial. Rev Eletron Enferm[Internet]. 2015[cited 2017 Aug 15]:17(2):322-32. Available from: https://www.revistas.ufg.br/fen/article/view/29570/19583

25. Knupp L. Motivação e desmotivação: desafio para as professoras doEnsino Fundamental. Educ. rev. [Internet]. 2006 [cited 2020Apr 12]; 27:77-290. Available from: <http://www.scielo.br/scielo.php?scrip$\mathrm{t}=\mathrm{sci}$ _arttext\&pid=S0104-40602006000100017\&lng=en\&nrm=iso>. ISSN 0104-4060. https://doi.org/10.1590/S010440602006000100017 .

26. Souza EFD, Silva AG, Silva AILF. Active methodologies for graduation in nursing: focus on the health care of older adults. Rev Bras Enferm [Internet]. 2018 [cited 2020 Apr 10];71(Suppl. 2):920-4. Available from: http://www.scielo.br/pdf/reben/v7ls2/0034-7167-reben-71-s2-0920.pdf.

27. Freire P. Pedagogia do oprimido. Rio de Janeiro: Paz e Terra; 2014.

28. Organização Mundial da Saúde. Global school health initiatives: achieving health and education outcomes. Report of a meeting. Bangkok, Thailand, 23-25 November 2015. Geneva: World Health Organization; 2017. [cited 2020 Apr 2]. Available from: https://www.who.int/ publications-detail/global-school-health-initiatives-achieving-health-and-education-outcomes

29. Teixeira EM, Machado MH, Medeiros HP. Tecnologias cuidativo-educacionais: uma possibilidade para o empoderamento do(a) enfermeiro(a)? Porto Alegre: Moriá; 2014.

30. Organização Mundial da Saúde (OMS). Relatório Mundial sobre a Prevenção da Violência 2014. Núcleo de Estudos da Violência (Trad.) São Paulo: OMS, 2015. [cited 2020 Apr 1]. Available from: https://nev. prp.usp.br/publicacao/relatorio-mundial-sobre-a-prevencao-da-violencia-2014/.

31. Ministério da Saúde (BR). Secretaria de Vigilância em Saúde. Departamento de Vigilância das Doenças Transmissiveis. Coordenação Geral de Hanseniase e Doenças em Eliminação. Brasilia, DF: Ministério da Saúde; 2018. [cited 2020 Apr 1]. Available from: http://portalarquivos2.saude.gov.br/images/pdf/2018/janeiro/31/2018004Hanseniase-publicacao.pdf. Acesso em: 06 dez. 2019. 